\title{
MFG-E8-mediated uptake of apoptotic cells by APCs links the pro- and antiinflammatory activities of GM-CSF
}

\author{
Masahisa Jinushi,1,2 Yukoh Nakazaki,,1,2 Michael Dougan,1,2 Daniel R. Carrasco,1,2,3 \\ Martin Mihm, ${ }^{4}$ and Glenn Dranoff ${ }^{1,2}$ \\ 1Department of Medical Oncology and Cancer Vaccine Center, Dana-Farber Cancer Institute, Boston, Massachusetts, USA. \\ 2Department of Medicine and 'Department of Pathology, Brigham and Women's Hospital and Harvard Medical School, Boston, Massachusetts, USA. \\ ${ }^{4}$ Department of Pathology, Massachusetts General Hospital and Harvard Medical School, Boston, Massachusetts, USA.
}

\begin{abstract}
Granulocyte-macrophage colony-stimulating factor (GM-CSF) enhances protection against tumors and infections, but GM-CSF-deficient mice develop inflammatory disease. Here we show that GM-CSF is required for the expression of milk fat globule EGF 8 (MFG-E8) in antigen-presenting cells, and that MFG-E8-mediated uptake of apoptotic cells is a key determinant of GM-CSF-triggered tolerance and immunity. Upon exposure to apoptotic cells, GM-CSF-deficient antigen-presenting cells (APCs) produce an altered cytokine profile that results in decreased Tregs and increased Th1 cells, whereas concurrent ablation of IFN- $\gamma$ promotes Th17 cells. In wild-type mice, MFG-E8 attenuates the vaccination activity of GM-CSF-secreting tumor cells through Treg induction, whereas a dominant-negative MFG-E8 mutant potentiates GM-CSF-stimulated tumor destruction through Treg inhibition. These findings clarify the immunoregulatory effects of apoptotic cells and suggest new therapeutic strategies to modulate $\mathrm{CD}^{+} \mathrm{T}$ cell subsets in cancer and autoimmunity.
\end{abstract}

\section{Introduction}

The mixture of cytokines produced in the tumor microenvironment plays a decisive role in determining the outcome of the host antitumor reaction (1). Cytokines released in response to cellular stress, injury, or infection stimulate the restoration of tissue homeostasis and restrict tumor development and progression. However, persistent cytokine expression in the setting of unresolved inflammation contributes to dysregulated cell growth and apoptosis and fosters tumor cell invasion and metastasis. This dual character of host immunity in cancer pathogenesis might reflect, at least in part, the pleiotropy of cytokine activities, with specific functions dependent upon the particular array of cells and soluble factors present in the tumor microenvironment.

Substantial evidence implicates an important role for granulocyte-macrophage colony-stimulating factor (GM-CSF) in antitumor responses. Vaccination with irradiated tumor cells engineered to secrete GM-CSF evokes potent, specific, and long-lasting antitumor immunity through improved tumor antigen presentation by dendritic cells and macrophages (2). Early-stage clinical testing of this immunization strategy revealed the consistent induction of antitumor cellular and humoral responses effectuating tumor necrosis in patients with advanced solid and hematologic malignancies (3). Additional tumor destruction may be accomplished in combination with antibody blockade of CTL-associated antigen-4 (CTLA-4) $(4,5)$. Notwithstanding this protective activity, other work indicates that tonic GM-CSF production by tumor cells may be associated with disease progression and that high levels of the

Nonstandard abbreviations used: CCL22, CC chemokine ligand 22; CTLA-4, CTL-associated antigen-4; Flt3-L, Flt3 ligand; Gas6, growth arrest-specific gene 6; GM-CSF, granulocyte-macrophage colony-stimulating factor; MFG-E8, milk fat globule EGF 8; TRP-2, tyrosinase related protein 2.

Conflict of interest: The authors have declared that no conflict of interest exists. Citation for this article: J. Clin. Invest. 117:1902-1913 (2007). doi:10.1172/JCI30966. cytokine may antagonize antitumor cytotoxicity $(6,7)$. Thus, the overall influence of GM-CSF during tumorigenesis might vary as a consequence of distinct secretion profiles and additional factors in the microenvironment.

As tumors arise from self, the impact of GM-CSF on host reactions might also involve a contribution of this cytokine to immune regulation. GM-CSF-deficient mice develop pulmonary alveolar proteinosis (PAP), a progressive accumulation of surfactant in lung air spaces, due to impaired alveolar macrophage differentiation, which compromises surfactant clearance and catabolism (8-10). This pulmonary pathology includes an extensive lymphoid hyperplasia circumscribing pulmonary airways and veins and reflects the absence of local GM-CSF production by type II pneumocytes (11).

Aged GM-CSF-deficient mice further manifest a SLE-like disorder characterized by immune complex deposition in the renal glomeruli and autoantibodies to double-stranded DNA and C1q (12). Mice doubly deficient in GM-CSF and IL-3, a closely related cytokine that signals through the shared $\beta_{\mathrm{c}}$ receptor subunit, similarly display PAP and SLE $(12,13)$. However, concurrent ablation of IFN- $\gamma$ in GM-CSF or GM-CSF/IL-3-deficient mice results in opportunistic infections and widespread inflammatory pathology, with acute and chronic granulomatous lesions present in multiple organs (12). Older compound cytokine-deficient animals succumb at high frequency to disseminated mature $B$ cell lymphomas and diverse solid neoplasms. The prophylactic administration of antibiotics suppresses the inflammatory pathology and subsequent tumor development, establishing the interplay of microbial agents and the loss of immune homeostasis as a critical determinant of tumor susceptibility. Together, these studies reveal a key role for GM-CSF individually and in combination with IL-3 and IFN- $\gamma$ in maintaining tolerance to self antigens and inhibiting tumor-promoting inflammation.

GM-CSF-deficient macrophages are impaired in the phagocytosis of apoptotic cells (12), a defect that might contribute to loss 
A

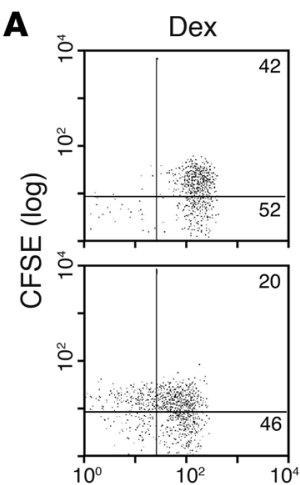

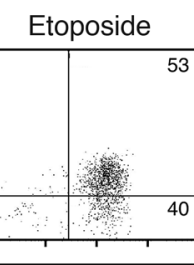

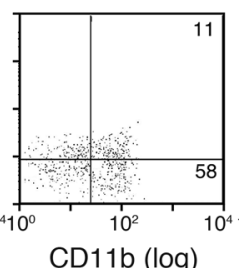

\section{Necrotic}
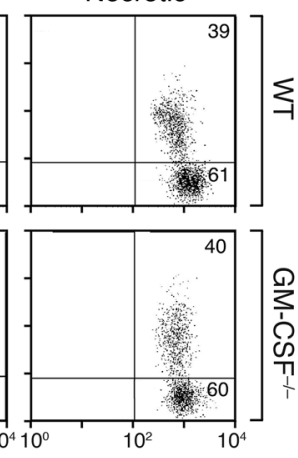

C
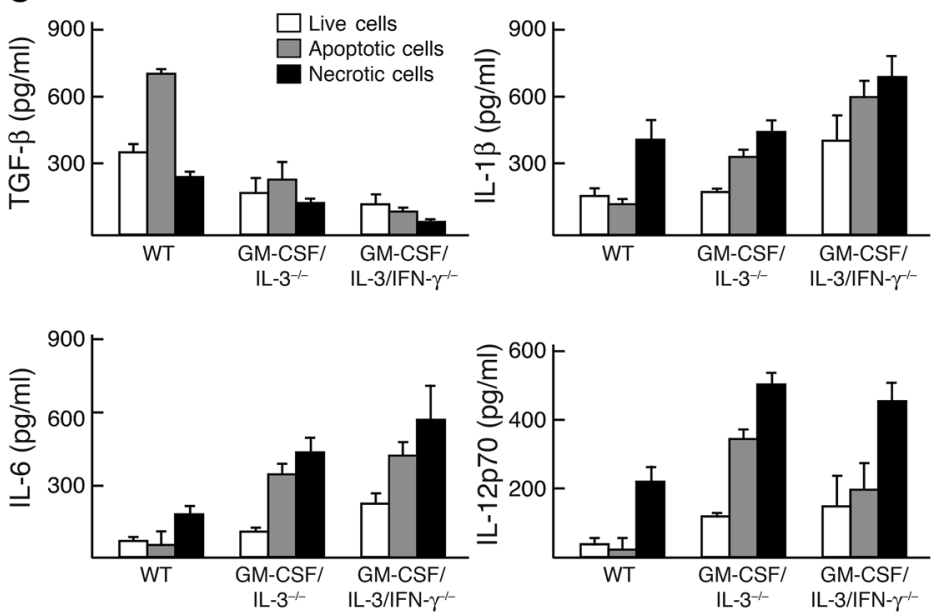

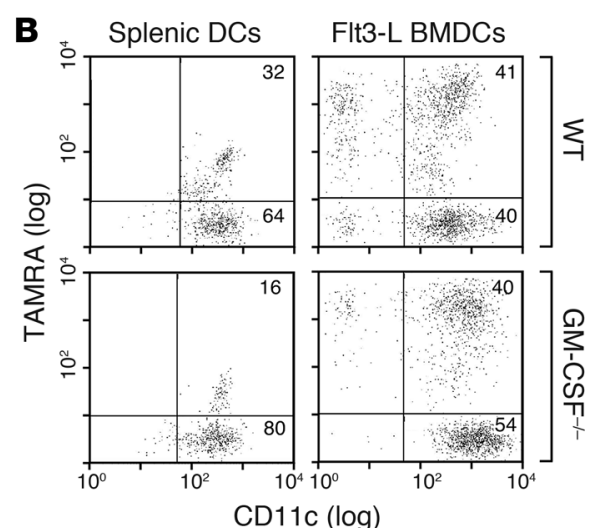

CD11c $(\log )$
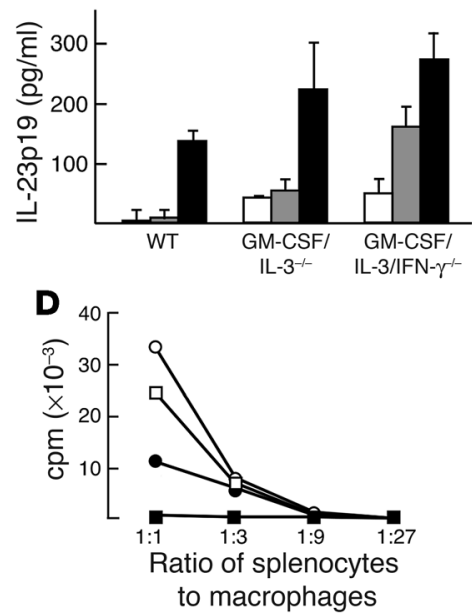

Figure 1

GM-CSF regulates the phagocytosis of apoptotic cells. (A) CFSE-labeled wild-type dying cells treated with dexamethasone (dex), etoposide, or necrotic cells were added to peritoneal macrophages, and phagocytosis was quantified by flow cytometry. Numbers refer to the percentage of cells within an indicated gate. (B) Purified splenic DCs or Flt3-L-derived bone marrow dendritic cells (BMDCs) were exposed to labeled apoptotic thymocytes, and phagocytosis was measured. (C) Peritoneal macrophages (3 mice per group) were loaded with apoptotic or necrotic thymocytes, and culture supernatants were measured by ELISA. (D) Peritoneal macrophages (circles, GM-CSF/IL-3/IFN- $\gamma$-deficient; squares, wild-type) were exposed to apoptotic (filled symbols) or necrotic (open symbols) thymocytes and cocultured with wild-type Balb/c splenocytes. Proliferation was determined by ${ }^{3} \mathrm{H}$-thymidine uptake. Results are representative of at least 2 or 3 independent experiments.

of immune regulation, as this activity normally stimulates antiinflammatory cytokines and inhibits proinflammatory mediators (14). Antigen-presenting cells exploit multiple receptors and secreted proteins to effect the clearance of dying cells (15). Among these, oxidized phosphatidylserine exposed on the surface of apoptotic cells constitutes a major signal for phagocyte engulfment. Milk fat globule EGF 8 (MFG-E8), Del-1, and growth arrest-specific gene 6 (Gas6) are secreted opsonins that bind phosphatidylserine and promote apoptotic cell ingestion through engagement of phagocyte $\alpha_{v} \beta_{3}$ and $\alpha_{v} \beta_{5}$ integrins and Mer receptor tyrosine kinase, respectively (16-18). Mice deficient in these pathways develop autoimmunity and persistent inflammation (19-21), although the underlying mechanisms remain to be fully defined.

The uptake of apoptotic cells by phagocytes may influence $\mathrm{CD} 4^{+}$ $\mathrm{T}$ cell function. In allogeneic bone marrow transplantation, the infusion of apoptotic cells stimulates the generation of FoxP3expressing Tregs through a pathway involving macrophages and TGF- $\beta$ production (22). In the autologous host, the ingestion of apoptotic neutrophils by tissue macrophages suppresses tonic IL-23 secretion and thereby modulates Th17 cells as part of a homeo- static circuit regulating granulopoiesis (23). Since Treg and Th17 cell development from a common $\mathrm{CD}^{+} \mathrm{T}$ cell precursor is influenced by TGF- $\beta$, IL-6, and other inflammatory mediators (24-26), the cytokine profile triggered by corpse clearance might contribute to $\mathrm{CD} 4^{+} \mathrm{T}$ cell differentiation.

Here we delineate critical roles for GM-CSF- and MFG-E8dependent uptake of apoptotic cells in regulating the balance of $\mathrm{CD} 4^{+} \mathrm{T}$ cell subsets in tolerance and tumor protection.

\section{Results}

GM-CSF regulates the phagocytosis of apoptotic cells. GM-CSF-deficient peritoneal macrophages manifest the impaired uptake of dexamethasone-treated wild-type apoptotic thymocytes (12). Similar defects were found with etoposide-exposed Jurkat $\mathrm{T}$ cells and $\gamma$-irradiated splenocytes, but the uptake of freeze-thaw-induced necrotic cells was intact (Figure 1A and data not shown). GM-CSF/IL-3- and GM-CSF/IL-3/IFN- $\gamma$-deficient macrophages displayed comparable phagocytosis profiles (data not shown). CD11c-expressing splenic dendritic cells were also impaired, although Flt3 ligand-derived (Flt3-L-derived) bone marrow dendritic cells efficiently ingested 

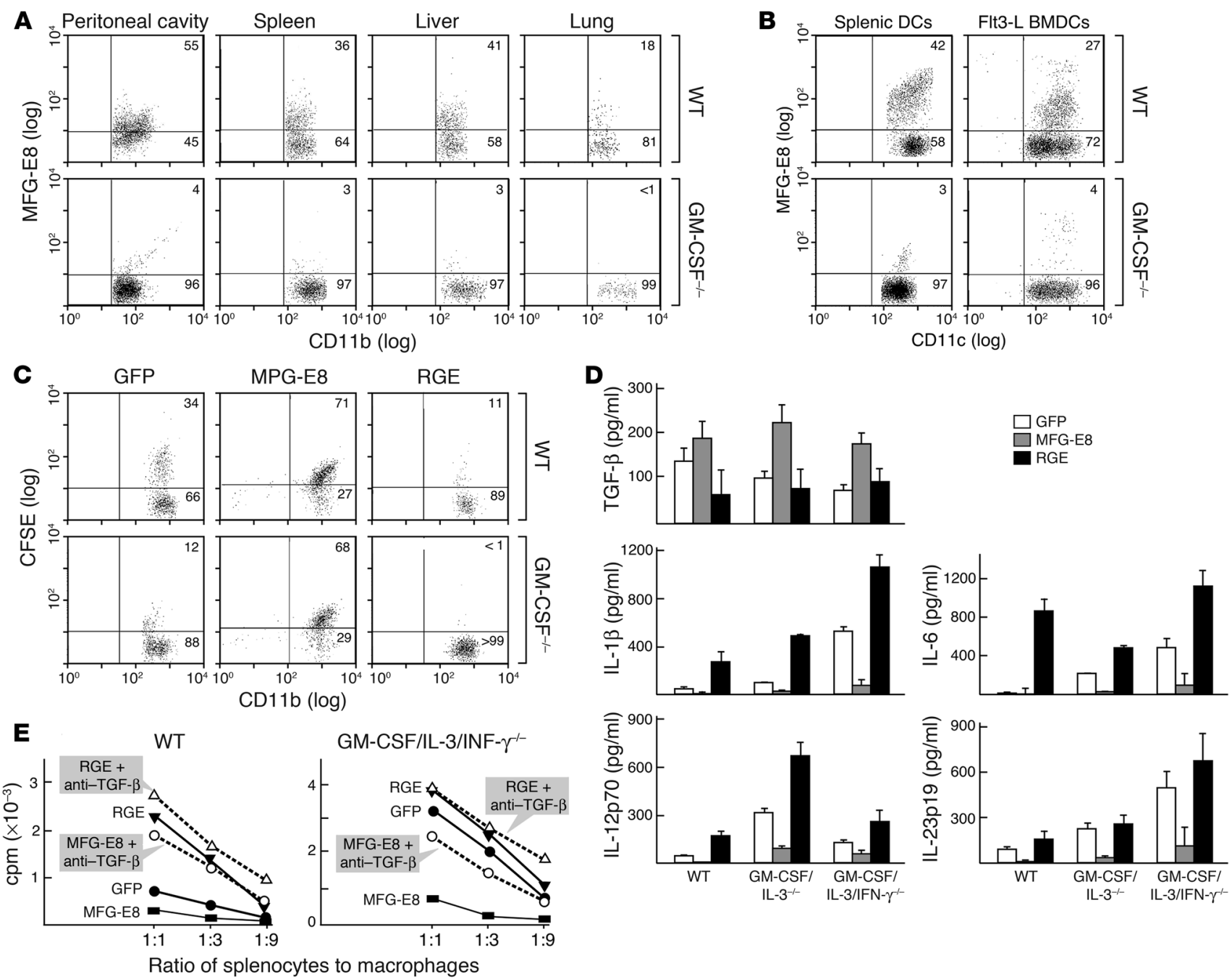

Figure 2

GM-CSF regulates MFG-E8-mediated uptake of apoptotic cells. (A) Purified macrophages were exposed to apoptotic cells overnight, and MFG-E8 expression was determined by flow cytometry. Numbers refer to the percentage of cells within an indicated gate. (B) Purified splenic dendritic cells or Flt3-L-derived bone marrow dendritic cells were exposed to apoptotic cells overnight and stained for MFG-E8. (C) Peritoneal macrophages were engineered to express MFG-E8, the RGE mutant, or GFP and were evaluated for the phagocytosis of labeled apoptotic thymocytes. (D) Transduced peritoneal macrophages (4 mice per group) were exposed to apoptotic thymocytes, and culture supernatants were analyzed by ELISA. (E) Transduced peritoneal macrophages were exposed to apoptotic thymocytes and then cocultured with wild-type Balb/c splenocytes with (open symbols) or without (filled symbols) neutralizing antibodies to TGF- $\beta$. Proliferation was determined by ${ }^{3} \mathrm{H}$-thymidine uptake. Results are representative of 2 to 4 independent experiments.

apoptotic material (Figure 1B). These results are in accord with a recent report indicating that Flt3-L-generated dendritic cells acquire dying cells through a "nibbling" process, in contrast to the engulfment that is triggered by GM-CSF (27). However, our findings suggest that GM-CSF may be a more important determinant of corpse clearance than Flt3-L under steady-state conditions.

As expected, wild-type macrophages triggered substantial TGF- $\beta$ but minimal IL-1 $\beta$, IL-6, IL-12p70, and IL-23p19 in response to apoptotic cells $(14,23,28)$, whereas necrotic cells evoked the opposite profile (Figure 1C). In contrast, GM-CSF- (data not shown), GM-CSF/IL-3-, and GM-CSF/IL-3/IFN- $\gamma$-deficient macrophages produced less TGF- $\beta$ but more IL- $1 \beta$, IL-6, IL-12p70, and IL-23p19 after exposure to apoptotic or necrotic cells. GM-CSF/ IL-3-deficient macrophages generated the highest IL-12p70, whereas GM-CSF/IL-3/IFN- $\gamma$-deficient macrophages generated the highest IL-23p19, indicating that IFN- $\gamma$ also contributes to cytokine regulation. IL-10 and TNF- $\alpha$ production did not differ across the set of mice (data not shown). While wild-type and GM-CSF/IL-3/IFN- $\gamma$-deficient macrophages loaded with necrotic cells stimulated robust allogeneic $\mathrm{T}$ cell responses, the ingestion of apoptotic cells by wild-type phagocytes suppressed proliferative responses more efficiently than GM-CSF/IL-3/IFN- $\gamma$-deficient phagocytes (Figure 1D).

GM-CSF regulates $M F G-E 8$ expression. Previous expression profiling experiments revealed that several gene products involved in the phagocytosis of apoptotic cells were induced in microglia with GM-CSF but not M-CSF (29). Real-time PCR analysis of GM-CSF-deficient peritoneal macrophages disclosed minimal 


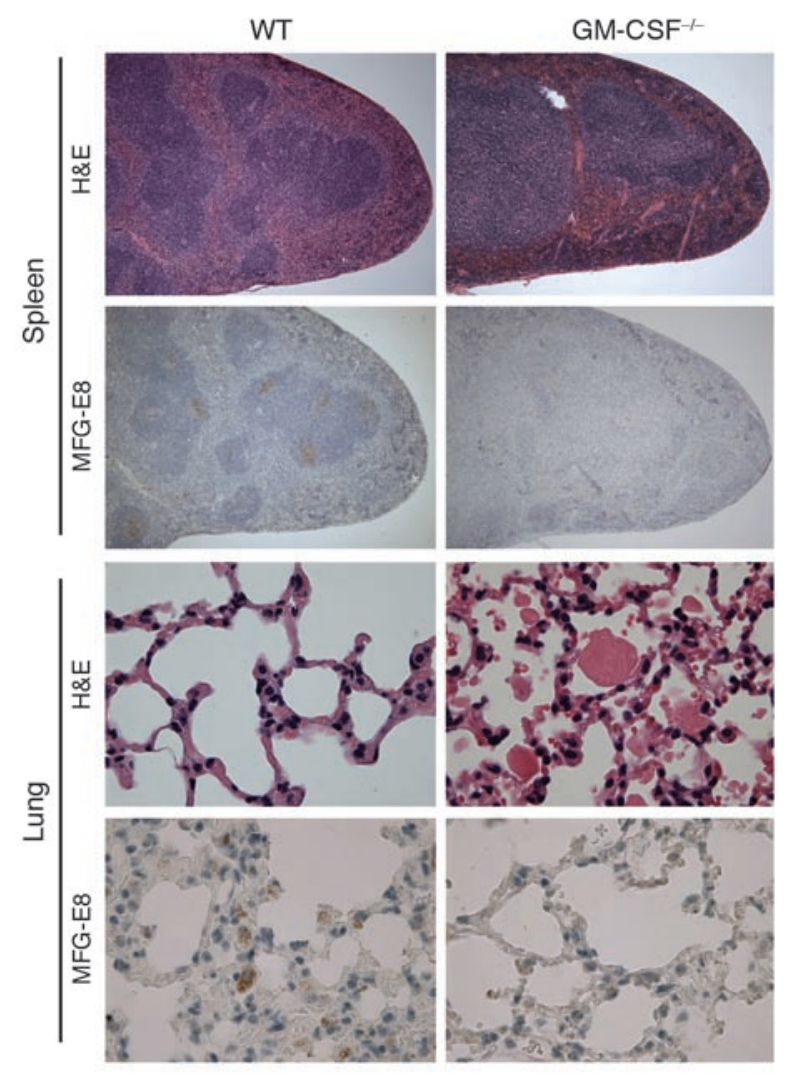

or no changes in the levels of MARCO, CD36, scavenger receptor-A, and the putative phosphatidylserine receptor, although MFG-E8 transcripts were significantly reduced compared with wild-type cells (data not shown). Flow cytometry confirmed the marked decrease in MFG-E8 expression in macrophages isolated from the peritoneal cavity, spleen, liver, and lung of GM-CSF-deficient mice (Figure 2A). While MFG-E8 was detected by immunohistochemistry in germinal center macrophages and alveolar macrophages of wild-type mice, consistent with previous reports (20), no staining was found in the spleens or lungs of GM-CSF-deficient animals (Figure 3) or in thymic macrophages from either strain (Supplemental Figure 1; supplemental material available online with this article; doi:10.1172/JCI30966DS1). Splenic dendritic cells and Flt3-L-derived bone marrow dendritic cells from GM-CSF-deficient mice also showed reductions in MFG-E8 compared with wild-type levels (Figure 2B), while similar decreases were observed with GM-CSF/IL-3 and GM-CSF/IL-3/ IFN- $\gamma$-deficient antigen-presenting cells (data not shown). Modest reductions in $\alpha_{v} \beta_{5}$, Gas6, and Mer were further detected (Supplemental Figure 2), suggesting that GM-CSF broadly regulates phosphatidylserine-based uptake of apoptotic cells.

To clarify the contribution of MFG-E8 deficiency to the impaired uptake of apoptotic cells, we used retroviral transduction to reconstitute MFG-E8 expression in peritoneal macrophages in vitro. A high-titer virus encoding a previously described MFG-E8 mutant, in which the RGD sequence involved in integrin binding was modified to RGE (30), was also generated. This protein retains the capacity to bind phosphatidylserine on apoptotic cells but cannot be internalized and thereby functions as a dominant-negative inhibitor. Flow cytometry documented that transduced cytokine-

\section{Figure 3}

GM-CSF regulates steady-state MFG-E8 expression in antigen-presenting cells in vivo. Anti-MFG-E8 staining was detected in germinal center macrophages and pulmonary alveolar macrophages of wildtype but not GM-CSF-deficient mice. The eosinophilic material in the alveoli of GM-CSF-deficient mice is pulmonary surfactant. Original magnification, $\times 25$ (spleen), $\times 250$ (lung).

deficient macrophages achieved MFG-E8 levels comparable to those of wild-type cells (data not shown). MFG-E8 restoration increased the phagocytosis of apoptotic cells in GM-CSF-deficient cells to wild-type levels, whereas MFG-E8 over-expression in wildtype cells further augmented corpse ingestion (Figure 2C). Confocal microscopy demonstrated that MFG-E8 mediated engulfment of apoptotic cells rather than simply mediating surface binding to phagocytes (Supplemental Figure 3). MFG-E8 levels did not alter the uptake of necrotic cells (data not shown). In contrast, the RGE mutant decreased apoptotic cell ingestion in wild-type and cytokine-deficient macrophages.

Enforced MFG-E8 expression in mutant macrophages restored TGF- $\beta$ and reduced IL-1 $\beta$, IL-6, IL-12p70, and IL-23p19 to levels comparable to wild-type controls (Figure 2D). Conversely, the RGE mutant decreased TGF- $\beta$ and increased IL-1 $\beta$, IL-6, IL-12p70, and IL-23p19 in wild-type macrophages. The RGE mutant also abrogated the inhibitory effects of apoptotic cells on the allostimulatory activity of wild-type peritoneal macrophages (Figure 2E), while MFG-E8 transduction normalized the aberrant allostimulatory activity of GM-CSF/IL-3/IFN- $\gamma$-deficient macrophages exposed to apoptotic cells. This suppression required TGF- $\beta$, as the addition of anti-TGF- $\beta$-neutralizing antibodies antagonized the effects of MFG-E8 but not those of the RGE mutant.

GM-CSF is required for Treg homeostasis through MFG-E8-mediated uptake of apoptotic cells. To explore whether the phagocytosis of apoptotic cells might influence Tregs, we cocultured syngeneic wild-type $\mathrm{CD}^{+} \mathrm{T}$ cells with apoptotic cell-loaded peritoneal macrophages and then analyzed FoxP3 expression (Figure 4A). Wildtype macrophages efficiently stimulated Tregs, but GM-CSF/ IL-3/IFN- $\gamma$ cells were impaired. MFG-E8 transduction of both wild-type and cytokine-deficient macrophages increased FoxP3 ${ }^{+}$ cells, although the RGE mutant decreased Tregs. Antibody blocking experiments established that MFG-E8-mediated Treg expansion required TGF- $\beta$ and MHC class II (Figure 4B). The FoxP3 ${ }^{+}$ cells proliferated during the coculture and manifested typical suppressor activity in allogeneic mixed leukocyte reactions (data not shown). Moreover, supernatants from apoptotic cell-loaded wild-type but not GM-CSF/IL-3/IFN- $\gamma$-deficient macrophages provoked selective migration of $\mathrm{CD} 4^{+} \mathrm{CD} 25^{+} \mathrm{T}$ cells in a CC chemokine ligand 22-dependent (CCL22-dependent) fashion (Figure 4C). Reconstitution of MFG-E8 expression in mutant macrophages restored chemotaxis (31).

Consistent with these results, the frequency of $\mathrm{CD} 3{ }^{+} \mathrm{CD} 4^{+} \mathrm{FoxP} 3^{+}$ $\mathrm{T}$ cells in GM-CSF- (data not shown), GM-CSF/IL-3- and GMCSF/IL-3/IFN- $\gamma$-deficient mice were significantly decreased compared with wild-type controls (Figure 4D). The absolute numbers of FoxP $3^{+}$cells were $3.5 \pm 1.8 \times 10^{5}$ (mean $\pm \mathrm{SD}$ ) cells/spleen for 5 wild-type mice, $0.7 \pm 0.1 \times 10^{5}$ cells/spleen for $5 \mathrm{GM}$-CSF/IL-3deficient mice, and $0.7 \pm 0.4 \times 10^{5}$ cells/spleen for 5 GM-CSF/IL-3/ IFN- $\gamma$-deficient mice $(P=0.007$, both knockout strains compared with wild-type). Similar Treg reductions were observed in lymph nodes but not thymi of mutant mice (Supplemental Figure 4), sug- 

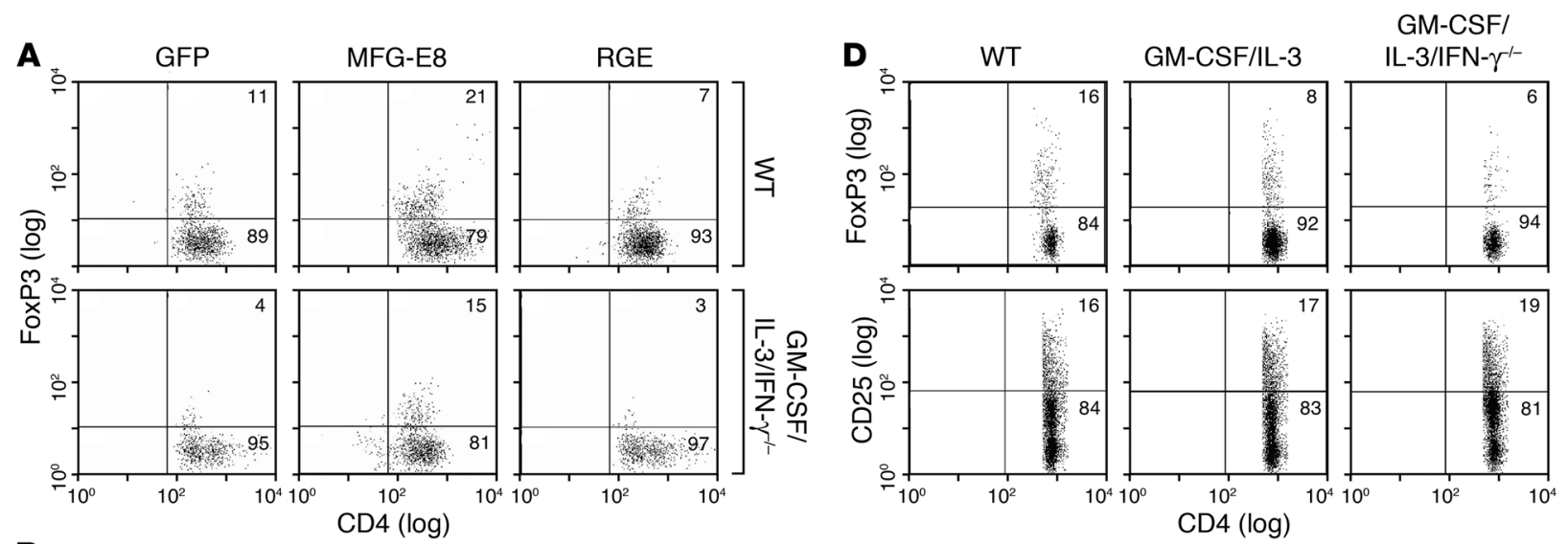

B
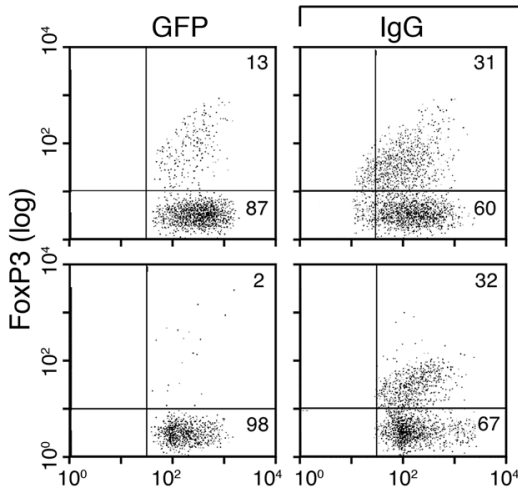

MFG-E8
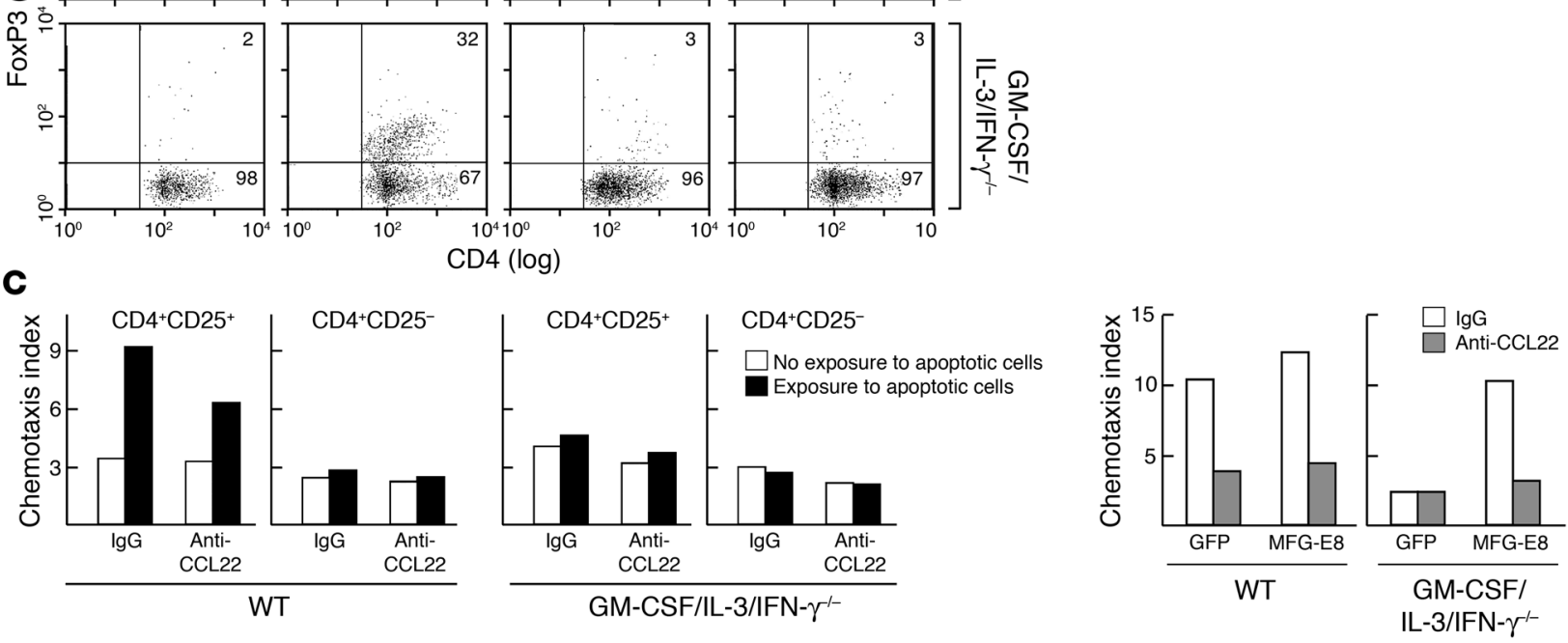

Figure 4

GM-CSF contributes to Treg homeostasis through MFG-E8. (A) Engineered peritoneal macrophages were exposed to apoptotic thymocytes and cocultured with wild-type syngeneic splenocytes. FoxP3-expressing Tregs were assayed by flow cytometry. Numbers refer to the percentage of cells within an indicated gate. (B) Blocking antibodies to TGF- $\beta$ or MHC class II were added to the coculture of apoptotic cell-loaded macrophages and syngeneic splenocytes. (C) Supernatants from macrophages exposed to apoptotic cells were assayed for chemotactic activity against $\mathrm{CD} 4{ }^{+} \mathrm{CD} 25^{+}$and CD4+CD25- T cells. Blocking antibodies against CCL22 or control isotype were added as indicated. (D) Splenocytes were analyzed for CD4, CD25, and FoxP3 expression. Results are representative of 2 to 6 experiments.

gesting that peripheral maintenance rather than production might be the primary defect. GM-CSF/IL-3/IFN- $\gamma$-deficient CD $4^{+} \mathrm{CD} 25^{+}$ $\mathrm{T}$ cells showed weaker suppressor activity compared with wildtype controls (Supplemental Figure 5), although additional studies using purified $\mathrm{FoxP}^{+}$cells are required to evaluate regulatory function on an individual cell basis.

$G M$-CSF regulates $C D 4^{+}$effector $T$ cell subsets through $M F G-E 8$. The increased production of inflammatory cytokines by GM-CSFdeficient antigen-presenting cells suggested that $\mathrm{CD} 4^{+}$effector $T$ cell subsets might be altered. We thus cocultured syngeneic wild-type $\mathrm{CD}^{+} \mathrm{CD} 45 \mathrm{RA}^{+} \mathrm{CD} 62^{\text {hi }}$ naive $\mathrm{T}$ cells with apoptotic cell-loaded peritoneal macrophages and analyzed IL-17 and
IFN- $\gamma$ expression by flow cytometry (Figure 5A). Consistent with the augmented IL-12p70 secretion, GM-CSF/IL-3-deficient macrophages stimulated increased Th1 cells. The combination of augmented IL-23, IL-1 $\beta$, and IL-6 levels in GM-CSF/IL-3/IFN- $\gamma-$ deficient macrophages yielded increased Th17 cells. MFG-E8 transduction of cytokine-deficient macrophages suppressed $\mathrm{CD}^{+}$effector cell responses. Comparable findings were obtained using Flt3-L-derived bone marrow dendritic cells (Figure 5B). Expression of the RGE mutant in wild-type macrophages promoted Th1 cells, whereas transduction of IFN- $\gamma$-deficient macrophages triggered Th17 cells (Figure 5C). Moreover, splenocytes stimulated with PMA and ionomycin showed Th1 skewing in 

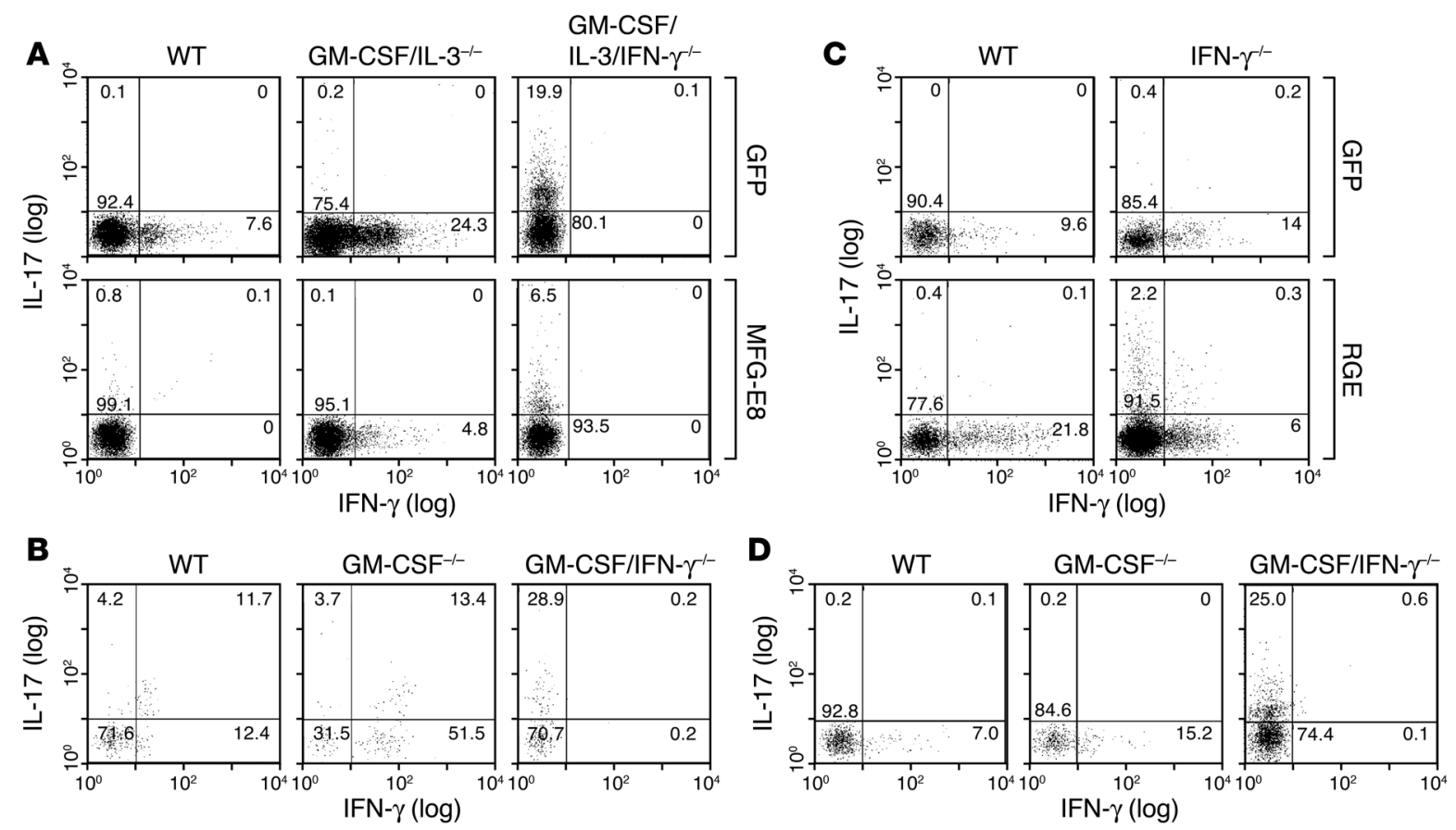

\section{Figure 5}

GM-CSF regulates CD4+ effector T cells through MFG-E8. (A) Peritoneal macrophages were exposed to apoptotic thymocytes and cocultured with syngeneic wild-type $C D 3^{+}$CD45RA ${ }^{+}$CD62 hi naive $T$ cells. T cells were then analyzed for IL-17 and IFN- $\gamma$ production. Numbers refer to the percentage of cells within an indicated gate. (B) Flt3-L-derived bone marrow dendritic cells were exposed to apoptotic thymocytes and used to stimulate syngeneic wild-type naive T cells. (C) Engineered macrophages were exposed to apoptotic thymocytes and used to stimulate syngeneic wild-type naive T cells. (D). Splenocytes were stimulated with PMA and ionomycin, and cytokine production of gated $\mathrm{CD}^{+} \mathrm{CD} 4^{+}$cells was determined by flow cytometry. Similar results were obtained in at least 2 experiments.

GM-CSF-deficient mice and Th17 skewing in GM-CSF/IFN- $\gamma$ deficient mice (Figure 5D).

$M F G-E 8$ reconstitution normalizes $C D 4^{+}$subsets in $G M-C S F$-deficient mice. To test further the role of MFG-E8 in GM-CSF-dependent responses in vivo, we performed bone marrow transplant experiments. Hematopoietic progenitors from wild-type and GM-CSF/ IL-3/IFN- $\gamma$-deficient mice were transduced with retroviral vectors and infused into lethally irradiated 2- to 4-month-old recipients. Mice were sacrificed 2 months after transplant, and GFP/MFG-E8 expression was documented in macrophages (data not shown). MFG-E8 increased phagocytosis of apoptotic cells in wild-type and GM-CSF/IL-3/IFN- $\gamma$-deficient macrophages, whereas the RGE mutant reduced corpse clearance in both strains (Figure 6A). MFG-E8 expression increased TGF- $\beta$ and reduced IL-6, IL-23p19, and IL-17 levels in the sera of wild-type and GM-CSF/IL-3/IFN- $\gamma$ deficient mice (Figure 6B). In contrast, the RGE mutant decreased TGF- $\beta$ but increased IL-6, IL-23p19, IL-17, and IL-12p70 levels in the sera of both strains. The RGE mutant also evoked circulating IFN- $\gamma$ in wild-type mice. This increase in proinflammatory cytokines might account for the low incidence of peri-transplant mortality observed only in animals that received RGE-expressing bone marrow (data not shown). Flow cytometry revealed that MFG-E8 increased FoxP3 ${ }^{+}$Tregs in wild-type and GM-CSF/IL-3/ IFN- $\gamma$-deficient splenocytes, whereas the RGE mutant decreased Tregs in wild-type mice (Figure 6C). MFG-E8 suppressed, but the RGE mutant increased Th17 cells in GM-CSF/IL-3/IFN- $\gamma$-deficient mice, while the RGE mutant augmented Th 1 cells in wildtype recipients (Figure 6D). MFG-E8 also restored the immunosup- pressive effects of apoptotic cells in GM-CSF/IL-3/IFN- $\gamma$-deficient mice, as documented by the reduced allostimulatory activity (Figure $6 \mathrm{E}$ ). Taken together, these findings establish a requirement for MFG-E8 in the immunoregulatory activities of GM-CSF.

Antigen-presenting cell maturation involves $M F G-E 8$ downregulation. Since MFG-E8 plays a critical role in GM-CSF-triggered tolerance, GM-CSF-induced protective immunity might involve downregulation of MFG-E8 function. Indeed, previous work showed robust MFG-E8 expression in immature, GM-CSF-derived bone marrow dendritic cells, whereas LPS-evoked maturation decreased MFG-E8 (32). Here we found that wild-type peritoneal macrophages treated with diverse TLR agonists including peptidoglycan (TLR2), poly-I-C (TLR3), LPS (TLR4), or CpG oligonucleotides (TLR9) suppressed MFG-E8 induction upon exposure to apoptotic cells (Figure 7A). Enforced MFG-E8 expression antagonized the reduction in TGF- $\beta$ and increase in IL- 6 stimulated by LPS (Figure 7B) and suppressed the enhanced allostimulatory activity of apoptotic cell-loaded macrophages treated with LPS (Figure 7C).

B220-, but not B220+, Flt3-L-derived bone marrow dendritic cells efficiently cross-present apoptotic cell antigens to stimulate $\mathrm{CD}^{+}$ cytotoxic T cell responses (27). MFG-E8 expression was restricted to $\mathrm{B} 220^{+}$dendritic cells, whereas maturation with LPS or necrotic cells downregulated MFG-E8 in these and GM-CSF-derived dendritic cells (Figure 7D). Sorted B220+, but not B220-, Flt3-Lderived dendritic cells secreted TGF- $\beta$ upon exposure to apoptotic cells but not necrotic cells (Figure 7E). In contrast, B220-, but not $\mathrm{B} 220^{+}$, dendritic cells produced IFN- $\alpha$ in response to apoptotic cells, and this was increased with LPS, as expected (27). B220-, 

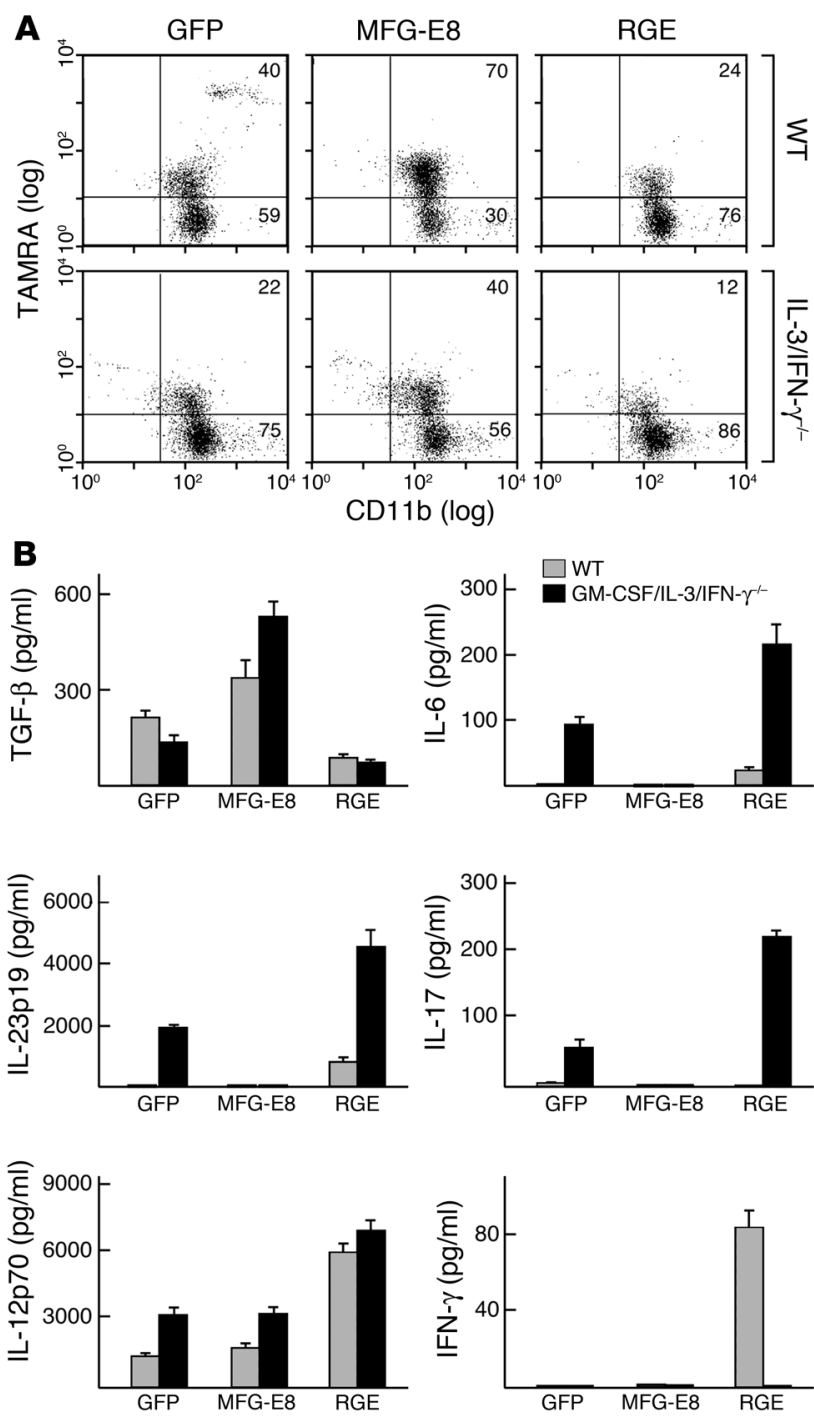
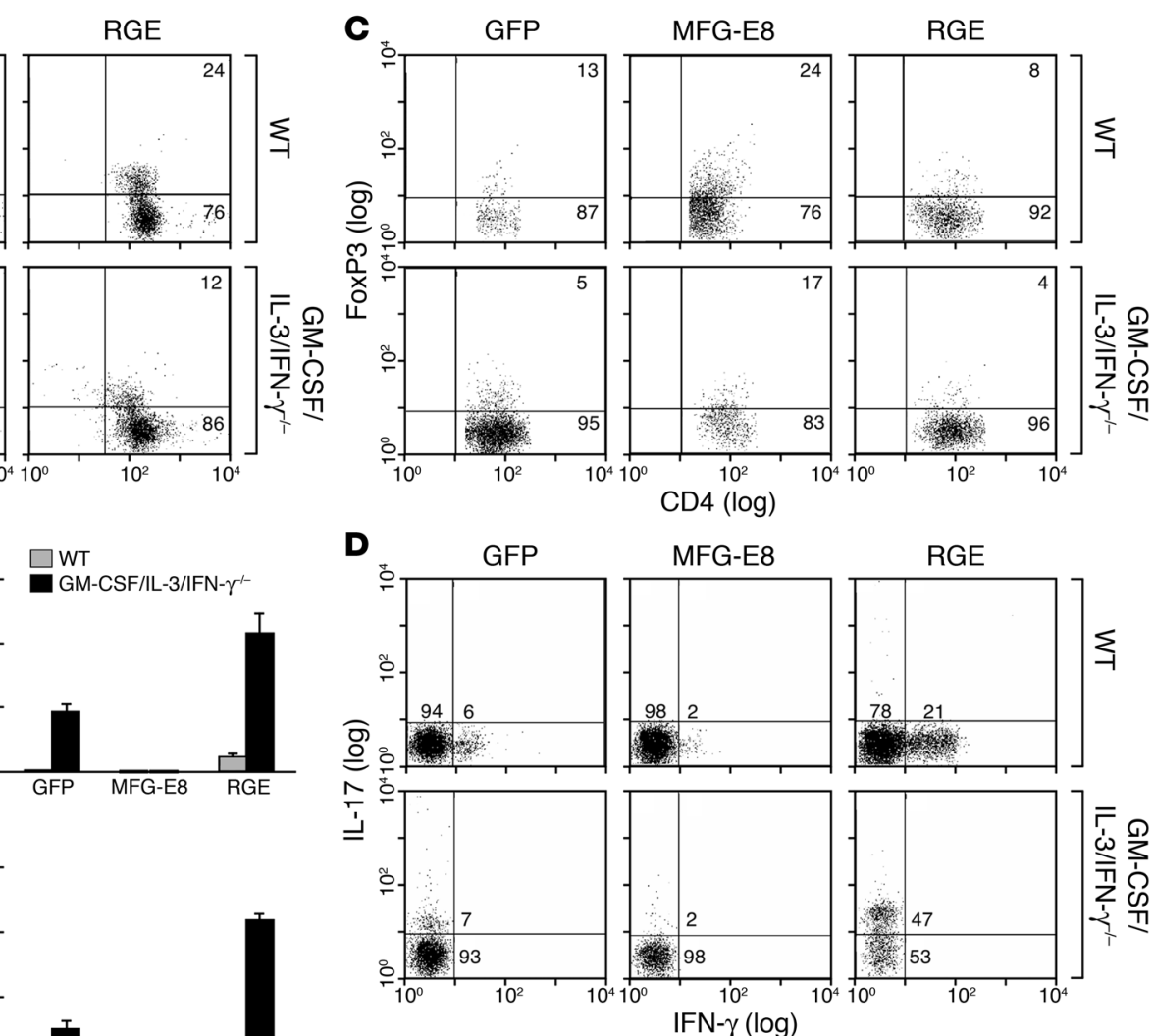

$\mathbf{E}$

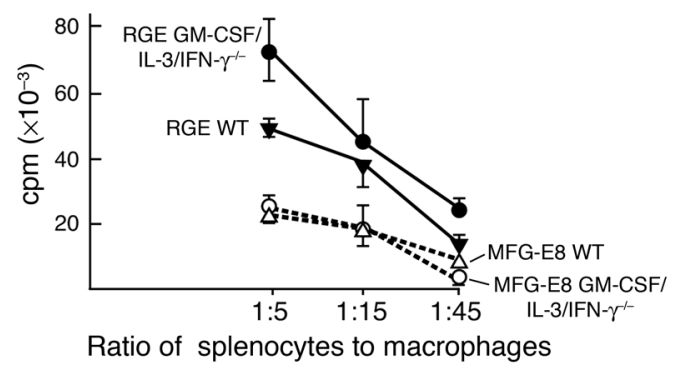

\section{Figure 6}

MFG-E8 reconstitution restores CD4+ T cell homeostasis in vivo. (A) Peritoneal macrophages recovered 2 months following transplantation (5 mice per group) were assayed for phagocytosis of labeled apoptotic thymocytes. Numbers refer to the percentage of cells within an indicated gate. (B) Serum cytokine levels measured by ELISA 2 months after transplant $(n=4)$. (C) Splenocytes $(n=4)$ were harvested 2 months after transplant and assayed for FoxP3 expression and (D) IL-17 and IFN- $\gamma$ expression (CD ${ }^{+}$CD $4{ }^{+}$gated cells). (E) Peritoneal macrophages from mice that received transplants were loaded with apoptotic cells and used to stimulate allogeneic Balb/c splenocytes. Proliferation was determined by ${ }^{3} \mathrm{H}$-thymidine uptake. Similar results were obtained with 2 independent transplant experiments.

but not $\mathrm{B} 220^{+}$, dendritic cells also produced IFN- $\alpha$ in response to necrotic cells. These results, together with our observation that Flt3-L-derived dendritic cells can ingest apoptotic cells through an MFG-E8 independent pathway (Figure 1B), support the idea that MFG-E8 expression in particular dendritic cell subsets is linked to the induction of tolerance rather than protective immunity.

$M F G-E 8$ regulates the antitumor effects of $G M-C S F-$ secreting vaccines. To examine the impact of MFG-E8 on GM-CSF-stimulated protective responses, we utilized the B16 melanoma model (2). In this system, vaccination with irradiated, GM-CSF-secreting tumor cells protected wild-type syngeneic C57BL/6 mice from subsequent challenge with live wild-type B16 cells, whereas vaccination with irradiated, parental B16 cells was ineffective (Figure 8A). Immunization with B16 cells expressing the RGE mutant failed to protect against tumor challenge, indicating that blockade of phosphatidylserine was not sufficient for protective immunity in this system. Vaccines composed of B16 cells secreting MFG-E8 were similarly inactive. However, coexpression of MFG-E8 abrogated the protective immunity elicited with GM-CSF-secreting tumor cells, whereas the RGE mutant did not.

To determine whether the RGE mutant might augment the antitumor effects of GM-CSF, we utilized a therapy model in which vaccination was begun on the same day as tumor challenge (Figure $8 \mathrm{~B})$. Under these conditions, irradiated, GM-CSF-secreting B16 cells evoked a modest delay in tumor growth, but all animals eventually succumbed to progressive tumor. MFG-E8 coexpression also inhibited the impact of GM-CSF-secreting tumor cells in this system. Nonetheless, the RGE mutant potentiated GM-CSF-induced 
A
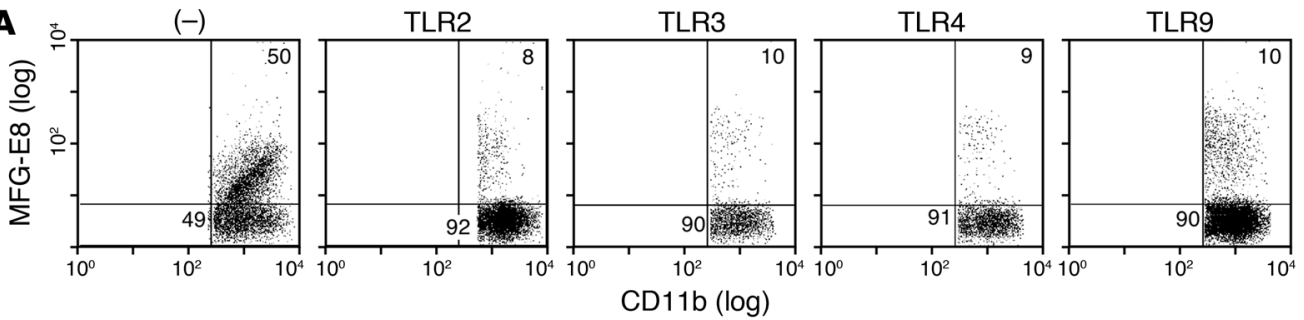

B

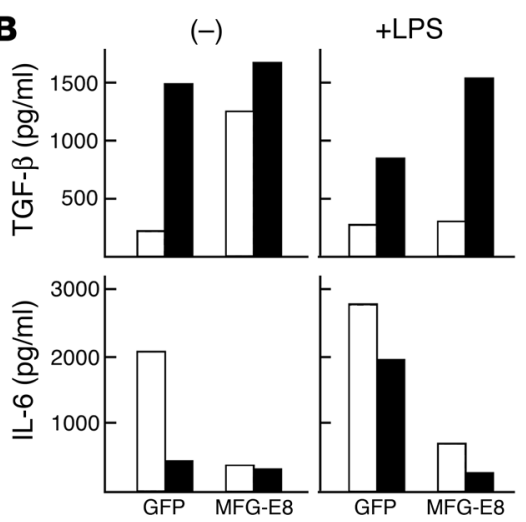

Exposure to apoptotic cells

$\square$ No exposure to apoptotic cells

C

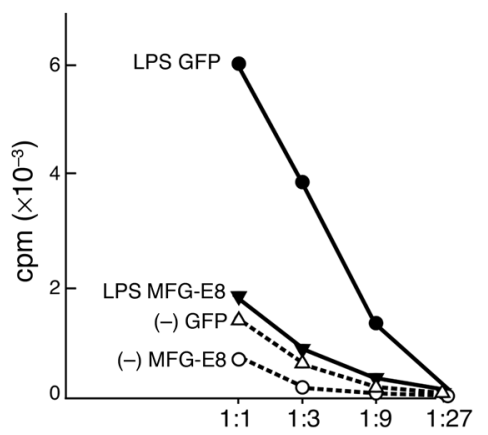

Ratio of splenocytes to macrophages
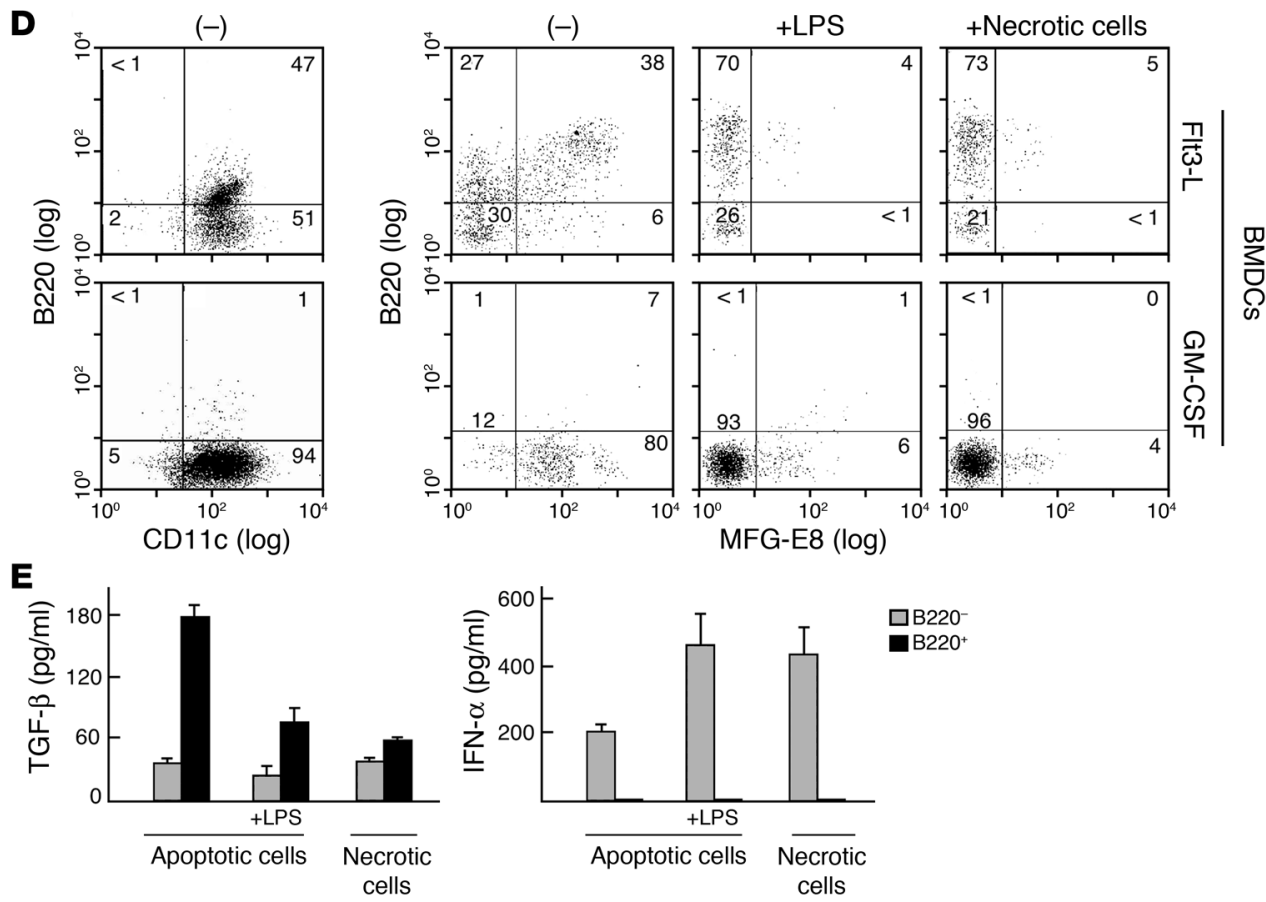

tumor destruction; when therapy was initiated 3 days after B16 injection, tumors grew initially, but the combination therapy effected complete tumor regressions (data not shown). No toxicities of treatment were observed.

To analyze the mechanisms underlying these effects, we isolated tumor-infiltrating lymphocytes from wild-type B16 challenge sites. The coexpression of MFG-E8 resulted in increased intratumoral Tregs (Figure 8C), whereas the coexpression of the RGE mutant inhibited Treg recruitment compared with B16 cells secreting only GM-CSF (Figure 8D). The RGE mutant also enhanced the activation of $\mathrm{CD}^{+}$tumor-infiltrating lymphocytes and increased the numbers of MHC class I restricted, tyrosinase related protein 2-

\section{Figure 7}

MFG-E8 expression is downregulated upon antigen-presenting cell maturation. (A) Wild-type peritoneal macrophages were exposed to apoptotic cells and peptidoglycan (TLR2), poly-IC (TLR3), LPS (TLR4), or CpG (TLR9), and MFG-E8 expression was determined. Numbers refer to the percentage of cells within an indicated gate. (B) Engineered wild-type peritoneal macrophages were exposed to apoptotic cells with or without LPS, and supernatants were assayed by ELISA. (C) Transduced peritoneal macrophages were exposed to apoptotic cells with or without LPS and cocultured with allogeneic Balb/c splenocytes. Proliferation was determined by ${ }^{3} \mathrm{H}$-thymidine uptake. (D) Flt3-L- or GMCSF-derived bone marrow dendritic cells were assayed for B220 and MFG-E8 expression. LPS or freeze-thaw-induced necrotic cells were added where indicated. (E) Flt3-L-derived dendritic cells were sorted into $\mathrm{B}^{2} 20^{+}$and $\mathrm{B}^{2} 20^{-}$populations, exposed to apoptotic or necrotic cells, and assayed for cytokine production by ELISA. Similar results were obtained in at least 2 independent experiments. -, no TLR agonist added. 
A

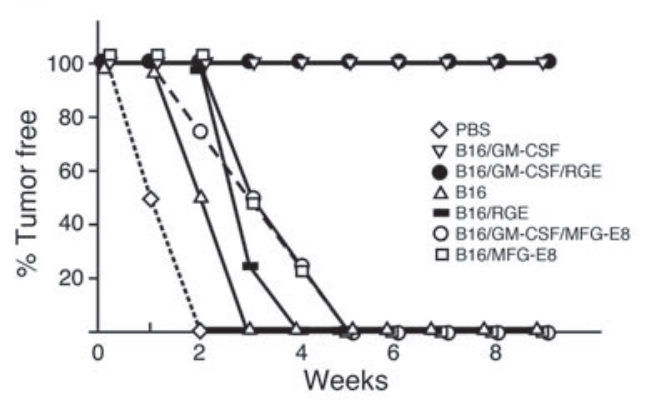

B

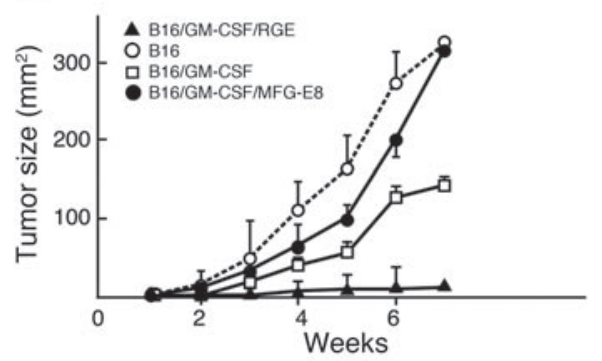

E

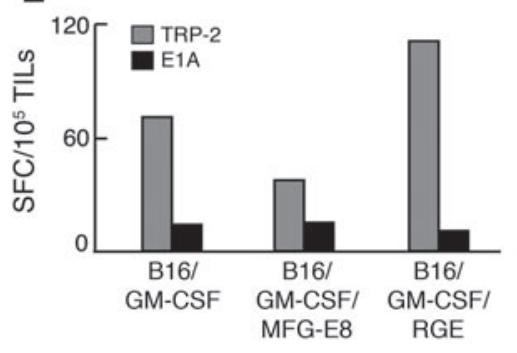

C
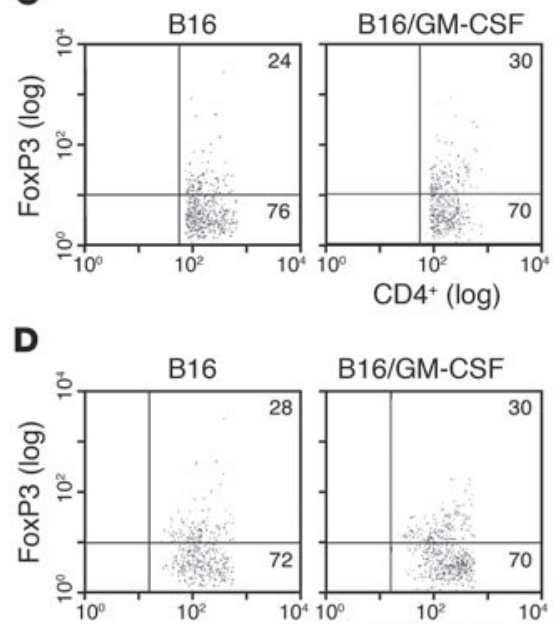

B16/GM-CSF

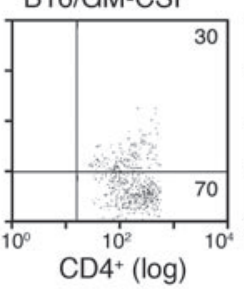

B16/GM-CSF/
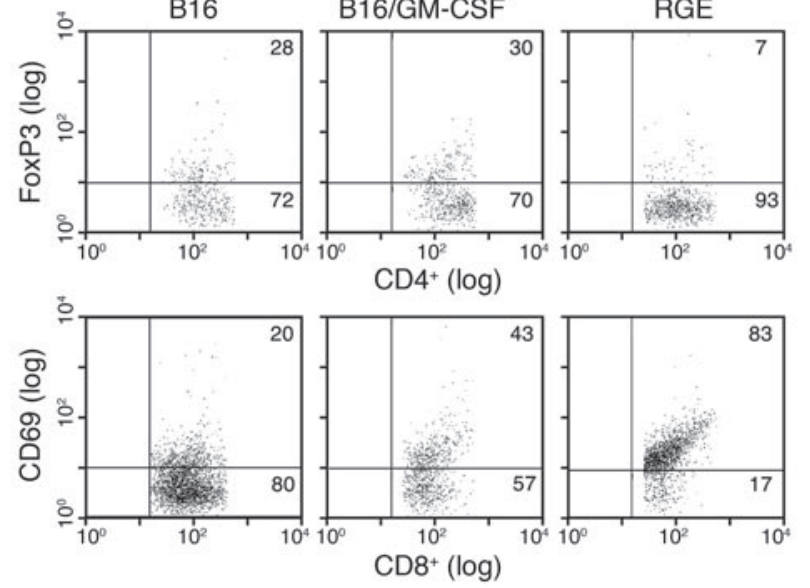

B16/GM-CSF/ MFG-E8

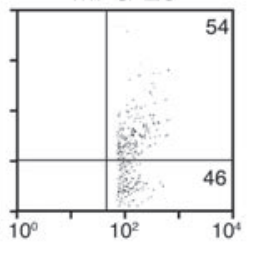



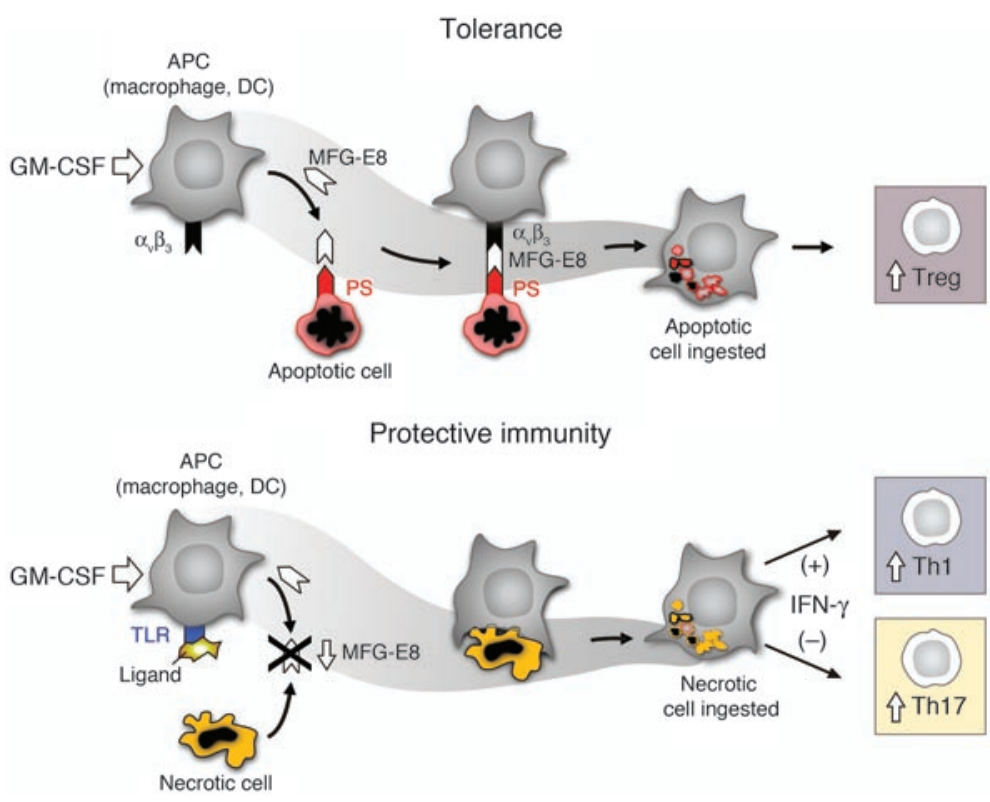

\section{Figure 9}

Proposed model of the dual roles for GM-CSF in tolerance and protective immunity. Top: GM-CSF is required for the expression of MFG-E8 in antigen-presenting cells. Upon exposure of phosphatidylserine (PS) on the surface of apoptotic cells, MFG-E8 promotes their engulfment by antigen-presenting cells, which results in the stimulations of Tregs. Bottom: In the setting of stress, TLR agonists and necrotic cells downregulate MFG-E8 on antigen-presenting cells, whereby GM-CSF promotes CD4+ effector T cell responses.

In light of the immunoregulatory activities of MFG-E8, why does the provision of GM-CSF as cancer therapy increase antitumor responses? Our results indicate that diverse TLR agonists and necrotic cells downregulate the expression of MFG-E8 on antigen presenting cells, and this suppression may be required for protective immunity. Indeed, enforced production of MFG-E8 antagonized the immunostimulatory impact of TLR agonists and irradiated, GM-CSF-secreting tumor cell vaccines, whereas high levels of endogenous MFG-E8 were associated with tolerogenic dendritic cell subsets. While blockade of MFG-E8 function with the RGE mutant was insufficient to evoke protection in the B16 melanoma model, in conjunction with GM-CSF, the strategy proved therapeutic against preexisting lesions. This intensified activity suggests that irradiated GM-CSF-secreting tumor vaccines alone trigger only partial downregulation of MFG-E8 in vivo. The enhanced immunity elicited by the combination treatment involved the inhibition of Tregs and the amplification of $\mathrm{CD}^{+}$cytotoxic $\mathrm{T}$ cells, resulting in a diversified host response capable of mediating regression of established tumors in the absence of toxicity. This mechanism of therapeutic synergy is distinct from that underlying CTLA-4 antibody blockade, which primarily targets effector cells but also increases Tregs (5). Thus, the 3 approaches together might prove complementary and achieve even higher levels of protective tumor immunity.

\section{Methods}

Mice. GM-CSF-, GM-CSF/IL-3-, IFN- $\gamma$ - (45), and GM-CSF/IL-3/IFN- $\gamma$ deficient mice were backcrossed at least 9 generations onto the C57BL/ 6 strain and housed under specific pathogen-free conditions. Genotypes were confirmed by PCR (12), and the experiments were approved by the Association for Assessment and Accreditation of Laboratory Animal Care International-accredited Dana-Farber Cancer Institute Institutional Animal Care and Use Committee.

Phagocytosis assays. Wild-type thymocytes were exposed to $10 \mu \mathrm{M}$ dexamethasone for 6 hours, splenocytes were exposed to 40 Gy $\gamma$-irradiation, and Jurkat $\mathrm{T}$ cells were exposed to $5 \mathrm{mg} / \mathrm{ml}$ etoposide for 16 hours to trigger apoptosis. Cell necrosis was induced with repetitive freeze-thaw cycles. Wild-type apoptotic or necrotic cells (scored with Annexin V and propidium iodide) were labeled with 5- (and 6-) carboxytetramethylrhodamine, succinimidyl ester (5[6]-TAMRA, SE; Invitrogen) or carboxyfluorescein diacetate succinimidyl ester (CFSE; Invitrogen) as described in ref. 12.

Thioglycollate-elicited peritoneal macrophages or CD11b microbeadpurified (Miltenyi Biotec) tissue macrophages were cultured in RPMI 1640 supplemented with $10 \%$ heat-inactivated fetal calf serum, L-glutamine, and penicillin/streptomycin. Dendritic cells were isolated from spleens using $\mathrm{CD} 11 \mathrm{c}$ microbeads (Miltenyi Biotec) or generated from bone marrow cells by culture for 7 days using conditioned media (added on days 0,2 , and 4) 
from B16 cells secreting GM-CSF or Flt3-L (46). Macrophages or dendritic cells were exposed to labeled apoptotic or necrotic cells and evaluated for phagocytosis efficiency by flow cytometry as previously reported (12).

Cytokine assays. TGF- $\beta$, IL-6, IL-1 $\beta$, IL-12p70, IL-23p19, IL-10, and TNF- $\alpha$ were measured in culture supernatants by ELISA, according to the manufacturer's directions (Pierce Biotechnology, R\&D Systems, eBioscience). For intracellular cytokine staining, T cells were stimulated with $1 \mathrm{mg} / \mathrm{ml}$ PMA and $50 \mathrm{ng} / \mathrm{ml}$ ionomycin; treated with GolgiPlug (BD Biosciences - Pharmingen); stained with anti-CD3, anti-CD4, or anti-CD8 mAbs; fixed and permeabilized with Cytofix/Cytoperm (BD Biosciences - Pharmingen); stained again with anti-IFN- $\gamma$, anti-IL-17, and isotype control mAbs (BD Pharmingen); and analyzed by flow cytometry.

Histology and immunohistochemistry. Formalin-fixed tissues were embedded in paraffin, sectioned, and stained with hematoxylin and eosin. Tissue sections were treated for antigen retrieval with a pressure cooker for 20 minutes and then incubated with $5 \mu \mathrm{g} / \mathrm{ml}$ of primary antibodies or a corresponding IgG fraction of preimmune serum in 3\% BSA/PBS blocking solution for 16 hours at $4^{\circ} \mathrm{C}$. The hamster anti-mouse MFG-E8 Ab (clone 18A-G10; MBL International) and mouse anti-human MFG-E8 Ab (clone 278918; R\&D Systems) were used. The primary antibodies were then visualized with the corresponding secondary biotinylated antibody and the streptavidin-peroxidase complex from Vector Laboratories.

Cell proliferation assays. Peritoneal macrophages were exposed to apoptotic or necrotic cells for 2 hours, washed, cocultured with Balb/c splenocytes for 72 hours, and ${ }^{3} \mathrm{H}$-thymidine uptake was determined. For suppression assays, $\mathrm{CD} 4{ }^{+} \mathrm{CD} 25^{+} \mathrm{T}$ cells were isolated from the splenocytes of wild-type and mutant mice with the regulatory $\mathrm{T}$ cell isolation kit (Miltenyi Biotec) following the manufacturer's instructions. Purified wild-type $\mathrm{CD} 4^{+} \mathrm{CD} 25$ splenic effector $\mathrm{T}$ cells were activated with $1 \mu \mathrm{g} / \mathrm{ml}$ plate-bound anti-CD3 $\mathrm{mAb}$ (clone 2C11; BD Biosciences - Pharmingen) in the presence or absence of regulatory $\mathrm{CD} 4^{+} \mathrm{CD} 25^{+} \mathrm{T}$ cells at various ratios for 72 hours, and proliferation was measured with ${ }^{3} \mathrm{H}$-thymidine incorporation. The suppressor activity was calculated as the percentage of proliferation measured in the cocultures versus effector cells only.

Chemotaxis assays. Supernatants of macrophages exposed to apoptotic cells were diluted 1:10 in culture media and added to the bottom wells of a microchamber containing an $8-\mu \mathrm{m}$ pore polycarbonate filter (CostarCorning). $\mathrm{CD} 4^{+} \mathrm{CD} 25^{+}$or $\mathrm{CD} 4^{+} \mathrm{CD} 25^{-} \mathrm{T}$ cells were applied to the upper chamber in the presence or absence of anti-human CCL22/MDC neutralizing $\mathrm{Ab}$ or isotype control (R\&D Systems), and the cells migrating to the bottom chamber were counted.

Flow cytometry. Macrophages were pretreated with GolgiPlug (BD Biosciences - Pharmingen), stained with anti-CD11b mAb (BD Biosciences - Pharmingen), fixed and permeabilized with Cytofix/Cytoperm buffer (BD Biosciences - Pharmingen), and stained again with unconjugated MFG-E8 $\mathrm{mAb}$ (Alexia) or Gas6 mAb (R\&D Systems) followed by PE-labeled goat antiIgG2 Ab (BD Biosciences - Pharmingen). For FoxP3 staining, lymphoid cells were labeled with anti-CD3 and CD4 mAbs (BD Biosciences - Pharmingen), washed, and then stained with PE-labeled anti-FoxP3 Ab using the FoxP3 staining set according to the manufacturer's protocol (eBioscience). Cell acquisition was performed with a FW501 flow cytometer (Beckman Coulter) and analyzed by FlowJo software (version 6.3.1; Tree Star).

Fluorescence microscopy. A total of $1 \times 10^{5}$ purified wild-type or mutant macrophages were stained with the green fluorescent cell linker reagent (Sigma-Aldrich), while $1 \times 10^{6}$ dexamethasone-treated wild-type thymocytes were stained with the red fluorescent cell linker reagent (Sigma-
Aldrich) according to the manufacturer's instructions. Apoptotic thymocytes were cocultured with macrophages for 6 hours on glass slides. The samples were then washed 3 times to remove floating cells, fixed with $20 \%$ methanol at $-20^{\circ} \mathrm{C}$ for 5 minutes, and visualized using a TE2000-U inverted fluorescence microscope (Nikon).

Retroviral mediated gene transfer. Full-length sequences encoding the open reading frames of MFG-E8 or the RGE mutant (which replaces the RGD sequence in the second EGF domain with RGE) were introduced into the PMFG retroviral vector and high-titer VSV-G-pseudotyped replication-defective viral stocks were prepared with 293-GPG cells as previously described (46). Peritoneal macrophages were induced to replicate by culture in DMEM plus $10 \%$ fetal calf serum supplemented with $10 \mathrm{ng} / \mathrm{ml}$ M-CSF (R\&D Systems). Viral supernatants were added to the cultured macrophages overnight in the presence of polybrene $(8 \mu \mathrm{g} / \mathrm{ml})$ to facilitate infection. The transduced cells were then washed and used for experiments $2-3$ days later.

After 48 hours of preconditioning with 5-fluorouracil $(150 \mathrm{mg} / \mathrm{kg})$, bone marrow cells were isolated from 8- to 10-week-old wild-type and GM-CSF/ IL-3/IFN- $\gamma$-deficient mice and cultured overnight with X-VIVO (Cambrex) supplemented with stem cell factor $(100 \mathrm{ng} / \mathrm{ml})$ and thrombopoietin $(50 \mathrm{ng} / \mathrm{ml})$. The cells were transduced with retroviral supernatants for 48 hours and then $1 \times 10^{6}$ cells were injected into lethally irradiated recipients ( 2 doses of 5.6 Gy, 6 hours apart, using a ${ }^{137}$ Cs source). Eight weeks after transplant, mice were sacrificed and analyzed for MFG-E8 expression, phagocytic capacity, and $\mathrm{CD} 4^{+} \mathrm{T}$ cell subsets as above.

B16 melanoma experiments. For tumor prevention studies, 8- to 12-weekold female C57BL $/ 6$ mice were injected subcutaneously in the flank with $1 \times 10^{6}$ irradiated (150 Gy) wild-type or retrovirally transduced (GM-CSF, MFG-E8, and/or RGE) B16 cells and 7 days later challenged subcutaneously on the back with $1 \times 10^{6}$ live B16 cells. For the therapy model, mice were injected on day 0 or 3 with $5 \times 10^{5}$ live B16 cells and treated on days 0 , 7 , and 14 with $1 \times 10^{6}$ irradiated, engineered B16 cells. Tumor growth was monitored, and the product of tumor diameters was recorded.

Tumor-infiltrating lymphocytes were obtained from B16 challenge sites using a Nocoprep (Axis-Shield) cell gradient separation followed by $\mathrm{CD}^{+}$ $\mathrm{T}$ cell purification with anti-CD3-labeled magnetic beads (Miltenyi Biotec). The cells were analyzed by flow cytometry using mAbs against CD4, CD8, $\mathrm{CD} 69$, and FoxP3. Antigen-specific $\mathrm{CD}^{+}$responses against $\mathrm{H}-2^{\mathrm{b}}$ restricted peptides derived from TRP-2 (180-188: SVYDFFVWL) or E1A (234-243: SGPSNTPPEI) were determined by incubating lymphocytes for 72 hours with $1 \times 10^{5} \mathrm{~B} 16$ cells and $25 \mathrm{U} / \mathrm{ml} \mathrm{IL}-2$ and measuring IFN- $\gamma$ production by ELISPOT using peptide-pulsed splenocytes as targets.

Statistics. A 1-sided exact Wilcoxon test was employed for statistical analysis.

\section{Acknowledgments}

We thank Tyler Hichman for help with the confocal microscopy. Supported by grants from the NIH and the Leukemia \& Lymphoma Society.

Received for publication November 15, 2006, and accepted in revised form April 10, 2007.

Address correspondence to: Glenn Dranoff, Dana-Farber Cancer Institute, Dana 520C, 44 Binney Street, Boston, Massachusetts 02115, USA. Phone: (617) 632-5051; Fax: (617) 632-5167; E-mail: glenn_dranoff@dfci.harvard.edu.
1. Dranoff, G. 2004. Cytokines in cancer pathogenesis and cancer therapy. Nat. Rev. Cancer. 4:11-22.

2. Dranoff, G., et al. 1993. Vaccination with irradiated tumor cells engineered to secrete murine granulocyte-macrophage colony-stimulating factor stimulates potent, specific, and long-lasting anti-tumor immunity. Proc. Natl. Acad. Sci. U. S. A. 90:3539-3543.
3. Hodi, F.S., and Dranoff, G. 2006. Combinatorial cancer immunotherapy. Adv. Immunol. 90:341-368. 4. Hodi, F.S., et al. 2003. Biologic activity of cytotoxic T lymphocyte-associated antigen 4 antibody block- 
ade in previously vaccinated metastatic melanoma and ovarian carcinoma patients. Proc. Natl. Acad. Sci. U. S. A. 100:4712-4717.

5. Quezada, S.A., Peggs, K.S., Curran, M.A., and Allison, J.P. 2006. CTLA4 blockade and GM-CSF combination immunotherapy alters the intratumor balance of effector and regulatory T cells. J. Clin. Invest. 116:1935-1945. doi:10.1172/JCI27745.

6. Sotomayor, E.M., et al. 1991. Role of tumor-derived cytokines on the immune system of mice bearing a mammary adenocarcinoma. II. Downregulation of macrophage-mediated cytotoxicity by tumorderived granulocyte-macrophage colony-stimulating factor. J. Immunol. 147:2816-2823.

7. Serafini, P., et al. 2004. High-dose granulocytemacrophage colony-stimulating factor-producing vaccines impair the immune response through the recruitment of myeloid suppressor cells. Cancer Res. 64:6337-6343.

8. Dranoff, G., et al. 1994. Involvement of granulocyte-macrophage colony-stimulating factor in pulmonary homeostasis. Science. 264:713-716.

9. Stanley, E., et al. 1994. Granulocyte/macrophage colony-stimulating factor-deficient mice show no major perturbation of hematopoiesis but develop a characteristic pulmonary pathology. Proc. Natl. Acad. Sci. U. S. A. 91:5592-5596.

10. Shibata, Y., et al. 2001. GM-CSF regulates alveolar macrophage differentiation and innate immunity in the lung through PU.1. Immunity. 15:557-567.

11. Huffman, J.A., Hull, W.M., Dranoff, G., Mulligan, R.C., and Whitsett, J.A. 1996. Pulmonary epithelial cell expression of GM-CSF corrects the alveolar proteinosis in GM-CSF-deficient mice. J. Clin. Invest. 97:649-655.

12. Enzler, T., et al. 2003. Deficiencies of GM-CSF and interferon-gamma link inflammation and cancer. J. Exp. Med. 197:1213-1219.

13. Gillessen, S., Mach, N., Small, C., Mihm, M., and Dranoff, G. 2001. Overlapping roles for granulocyte-macrophage colony-stimulating factor and interleukin-3 in eosinophil homeostasis and contact hypersensitivity. Blood. 97:922-928.

14. Fadok, V.A., Bratton, D.L., and Henson, P.M. 2001. Phagocyte receptors for apoptotic cells: recognition, uptake, and consequences. J. Clin. Invest. 108:957-962. doi:10.1172/JCI200114122.

15. Savill, J., Dransfield, I., Gregory, C., and Haslett, C. 2002. A blast from the past: clearance of apoptotic cells regulates immune responses. Nat. Rev. Immunol. 2:965-975

16. Hanayama, R., et al. 2002. Identification of a factor that links apoptotic cells to phagocytes. Nature. 417:182-187.
17. Hanayama, R., Tanaka, M., Miwa, K., and Nagata, S. 2004. Expression of developmental endothelial locus- 1 in a subset of macrophages for engulfment of apoptotic cells. J. Immunol. 172:3876-3882.

18. Ishimoto, Y., Ohashi, K., Mizuno, K., and Nakano, T. 2000. Promotion of the uptake of PS liposomes and apoptotic cells by a product of growth arrest-specific gene, gas6. J. Biochem. (Tokyo). 127:411-417.

19. Cohen, P.L., et al. 2002. Delayed apoptotic cell clearance and lupus-like autoimmunity in mice lacking the c-mer membrane tyrosine kinase. J. Exp. Med. 196: $135-140$

20. Hanayama, R., et al. 2004. Autoimmune disease and impaired uptake of apoptotic cells in MFGE8-deficient mice. Science. 304:1147-1150.

21. Scott, R.S., et al. 2001. Phagocytosis and clearance of apoptotic cells is mediated by MER. Nature. 411:207-211.

22. Kleinclauss, F., et al. 2006. Intravenous apoptotic spleen cell infusion induces a TGF-beta-dependent regulatory T-cell expansion. Cell Death Differ. 13:41-52.

23. Stark, M.A., et al. 2005. Phagocytosis of apoptotic neutrophils regulates granulopoiesis via IL-23 and IL-17. Immunity. 22:285-294.

24. Bettelli, E., et al. 2006. Reciprocal developmental pathways for the generation of pathogenic effector TH17 and regulatory T cells. Nature. 441:235-238.

25. Mangan, P.R., et al. 2006. Transforming growth factor-beta induces development of the $\mathrm{T}(\mathrm{H}) 17$ lineage. Nature. 441:231-234.

26. Veldhoen, M., Hocking, R.J., Atkins, C.J., Locksley, R.M., and Stockinger, B. 2006. TGFbeta in the context of an inflammatory cytokine milieu supports de novo differentiation of IL-17-producing T cells. Immunity. 24:179-189.

27. Janssen, E., et al. 2006. Efficient T cell activation via a toll-interleukin 1 receptor-independent pathway. Immunity. 24:787-799.

28. Kim, S., Elkon, K.B., and Ma, X. 2004. Transcriptional suppression of interleukin-12 gene expression following phagocytosis of apoptotic cells. Immunity. 21:643-653.

29. Re, F., et al. 2002. Granulocyte-macrophage colonystimulating factor induces an expression program in neonatal microglia that primes them for antigen presentation. J. Immunol. 169:2264-2273.

30. Asano, K., et al. 2004. Masking of phosphatidylserine inhibits apoptotic cell engulfment and induces autoantibody production in mice. J. Exp. Med. 200:459-467.

31. Curiel, T.J., et al. 2004. Specific recruitment of regulatory $\mathrm{T}$ cells in ovarian carcinoma fosters immune privilege and predicts reduced survival. Nat. Med. 10:942-949.

32. Miyasaka, K., Hanayama, R., Tanaka, M., and Nagata, S. 2004. Expression of milk fat globule epidermal growth factor 8 in immature dendritic cells for engulfment of apoptotic cells. Eur. J. Immunol. 34:1414-1422.

33. Dunn, G.P., Old, L.J., and Schreiber, R.D. 2004. The immunobiology of cancer immunosurveillance and immunoediting. Immunity. 21:137-148.

34. Burgess, A., Camarkis, J., and Metcalf, D. 1977. Purification and properties of colony-stimulating factor from mouse lung-conditioned medium. J. Biol. Chem. 252:1998-2003.

35. Fleetwood, A.J., Cook, A.D., and Hamilton, J.A. 2005. Functions of granulocyte-macrophage colony-stimulating factor. Crit. Rev. Immunol. 25:405-428.

36. Schwarzenberger, P., et al. 1998. IL-17 stimulates granulopoiesis in mice: use of an alternate, novel gene therapy-derived method for in vivo evaluation of cytokines. J. Immunol. 161:6383-6389.

37. Wiekowski, M.T., et al. 2001. Ubiquitous transgenic expression of the IL-23 subunit p19 induces multiorgan inflammation, runting, infertility, and premature death. J. Immunol. 166:7563-7570.

38. Langowski, J.L., et al. 2006. IL-23 promotes tumour incidence and growth. Nature. 442:461-465.

39. Numasaki, M., et al. 2003. Interleukin-17 promotes angiogenesis and tumor growth. Blood. 101:2620-2627.

40. Nelson, W.G., De Marzo, A.M., and Isaacs, W.B. 2003. Prostate cancer. N. Engl. J. Med. 349:366-381.

41. Gallina, G., et al. 2006. Tumors induce a subset of inflammatory monocytes with immunosuppressive activity on $\mathrm{CD}^{+} \mathrm{T}$ cells. J. Clin. Invest. 116:2777-2790.

42. Rabinovich, G.A., Gabrilovich, D., and Sotomayor, E.M. 2006. Immunosuppressive strategies that are mediated by tumor cells. Annu. Rev. Immunol. 25:267-296.

43. Dranoff, G. 2005. The therapeutic implications of intratumoral regulatory $\mathrm{T}$ cells. Clin. Cancer Res. 11:8226-8229.

44. Silvestre, J.S., et al. 2005. Lactadherin promotes VEGF-dependent neovascularization. Nat. Med. 11:499-506.

45. Dalton, D., et al. 1993. Multiple defects of immune cell function in mice with disrupted interferon- $\gamma$ genes. Science. 259:1739-1742.

46. Mach, N., et al. 2000. Differences in dendritic cells stimulated in vivo by tumors engineered to secrete granulocyte-macrophage colony-stimulating factor or flt3-ligand. Cancer Res. 60:3239-3246. 